\title{
Considerations for the Management of Cryptotia Based on the Experience of 34 Patients
}

\author{
Seok-Kwun Kim, Chung-Min Yoon, Myung-Hoon Kim, Min-Su Kim, Keun-Cheol Lee \\ Department of Plastic and Reconstructive Surgery, Dong-A University School of Medicine, Busan, Korea
}

Background Cryptotia is a congenital ear deformity in which the upper pole appears buried beneath the mastoid skin. Cryptotia is a common auricular malformation among Asians. The aim of this paper is to examine the surgical techniques for and complications of 34 cryptotic patients.

Methods Surgery was performed for 34 cryptotic deformities (January 2005 to January 2012). Twenty-two patients (64.7\%) were classified as having type I cryptotia, and 12 patients (37.5\%) type II cryptotia. Among the type I cryptotia patients, 8 patients had mild deformity and 14 severe deformity. Among the type II cryptotia patients, 10 patients had mild deformity and 2 severe deformity.

Results The mild deformities were corrected via Z-plasty, V-Y plasty, full-thickness skin graft, and transposition flap, while the severe deformities were corrected via cartilage graft or Medporfor the spread of cartilage adhesion of antihelix. There were two cases of reinvagination in the autologous cartilage graft group. Implant exposure occurred with Medpor (two cases). There were two cases of hypertrophic scar on the previous surgical wound with Medpor. There were no complications in the 18 patients who had mild deformities.

Conclusions The type I cryptotia patients had more severe deformities than the type II cryptotia patients. As most of the type II cryptotia patients had only mild deformities, their deformities were corrected without using autologous conchal cartilage graft or Medpor, except for two patients. Through more case analyses, researchers should make an effort to identify methods for recurrence and prevention of complication.

Keywords Classification / Cartilage / Medpor
Correspondence: Seok-Kwun Kim Department of Plastic and Reconstructive Surgery, Dong-A University School of Medicine, 26 Daesingongwon-ro, Seo-gu, Busan 602-715, Korea

Tel: $+82-51-240-2807$

Fax: +82-51-243-5416

E-mail:sgkim1@dau.ac.kr

This study was supported by research funds from Dong-A University.

This study was presented at the 69th Congress of the Korean Society of

Plastic and Reconstructive Surgeons on November 11-13, 2011 in Seoul, Korea.

No potential conflict of interest relevant to this article was reported.

\section{INTRODUCTION}

Cryptotia is a congenital ear deformity in which the upper pole appears buried beneath the mastoid skin. It is a common auricular malformation among Asians. The upper portion of the auricle is buried beneath the temporal skin and can be pulled out by the hand, but it returns to its original state when released [1]. This buried state is not cosmetically acceptable [1]. A cryptotia patient has difficulty wearing glasses or a mask. Hirose et al. [2] classified cryptotia into two types according to the type of antihelix deformity and the state of the intrinsic auricular muscle. In type I cryptotia, the body and superior crus of the antihelix are compressed together so that the upper portion is buried beneath the skin. In type II cryptotia, there is a gross contraction of the body of the antihelix, and the inferior crus of the antihelix is acutely bent over [2]. The authors appreciated the value of 
Hirose's cryptotia classification. Using Hirose's classification, the authors aimed to confirm that all cryptotic patients have different degrees of cartilage or skin deformation and aimed to develop an appropriate surgical method accordingly. From January 2005 to January 2012, surgery was performed on 34 cryptotic patients. The aim of this paper is to consider the surgical technique and complications of 34 cryptotic patients.

\section{METHODS}

\section{Subjects}

This study focused on patients who underwent cryptotia correction within the period from January 2005 to January 2012. Of the 34 patients between the ages of 1 and 46 years who were diagnosed with cryptotia, 22 were classified as having type I

\section{Fig. 1. Eight-year-old type II unilateral cryptotic patient}

(A) Preoperative view. (B) Immediate postoperative findings. Z-plasty was performed.
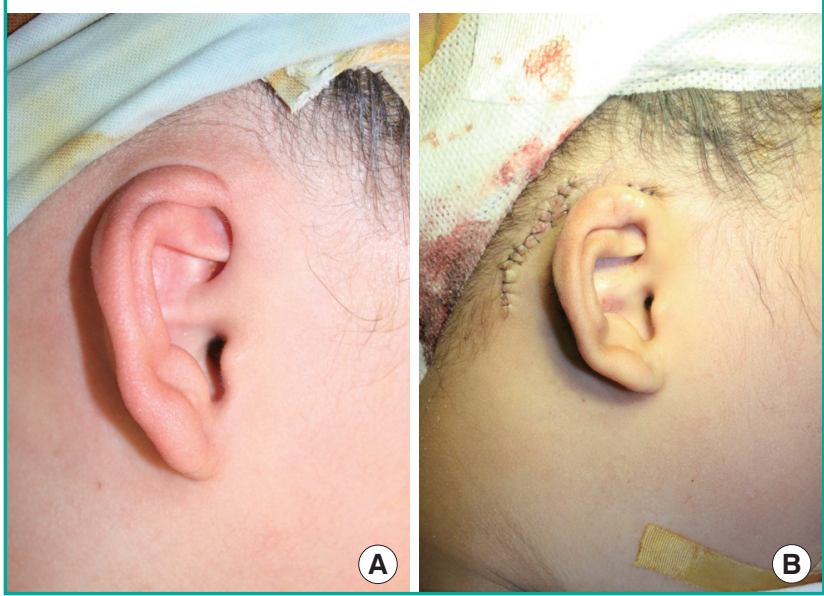

Fig. 2. Four-year-old type II unilateral cryptotic patient

(A) Preoperative view. (B) Findings at two months postoperatively. $\mathrm{V}-\mathrm{Y}$ plasty was performed.
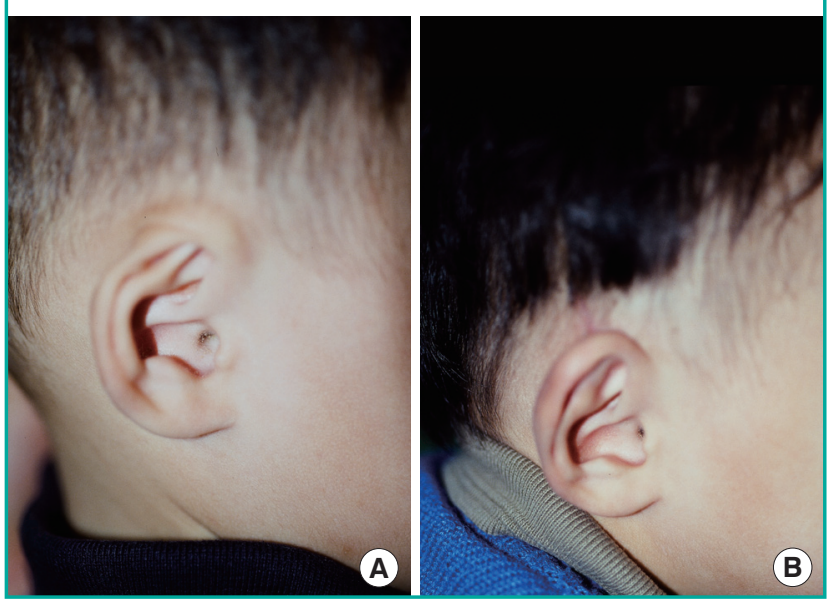

cryptotia (64.7\%), and 12 type II cryptotia (35.3\%). Among the type I cryptotia patients, 18 had unilateral cryptotia and 4 bilateral cryptotia while among the type II cryptotia patients, 10 had unilateral cryptotia and 2 bilateral cryptotia. Among the type I cryptotia patients, 8 had mild (skin) deformities and 14 severe (cartilage) deformities, while among the type II cryptotia patients, 10 had mild deformities and 2 severe deformities. In the severe cases, the cryptotic patients had severe degrees of skin or cartilage deformation and had high recurrent rate of invagination when the cartilage deformation was not corrected. Thus, the authors attempted various cartilage sutures, high-density polyethylene implants (Medpor, Portex Industries, Fairburn, GA, USA), and cavum concha cartilage grafting.

\section{Surgical techniques}

Among the 22 type I cryptotia patients, 8 patients had mild (skin) deformities. Of these, the deformities of four were corrected via Z-plasty (Fig. 1), those of 2 were corrected via V-Y plasty (Fig. 2), and those of the remaining two were corrected via full-thickness skin graft and transposition flap (Fig. 3). Fourteen patients had severe (cartilage) deformities. The Onizuka method was modified for skin flap (Fig. 4) [3]. The severe deformities of 10 patients were corrected using cartilage graft from the cavum concha, and those of 4 patients were corrected via antihelical adhesion through Medpor implantation after the detachment of the adhesion. Among the 12 type II cryptotia patients, 10 had mild deformities. Of these patients, the mild deformities of six were corrected via Z-plasty, and those of 4 were corrected via V-Y plasty. The severe deformities of 2 patients were corrected via the modified Onizuka method. The cartilage deformities were corrected via antihelical adhesion through Medpor implantation (Figs. 5, 6).

\section{Fig. 3. Six-year-old type I unilateral-cryptotic patient}

(A) Preoperative view. (B) Immediate postoperative findings. Fullthickness skin grafts was performed.
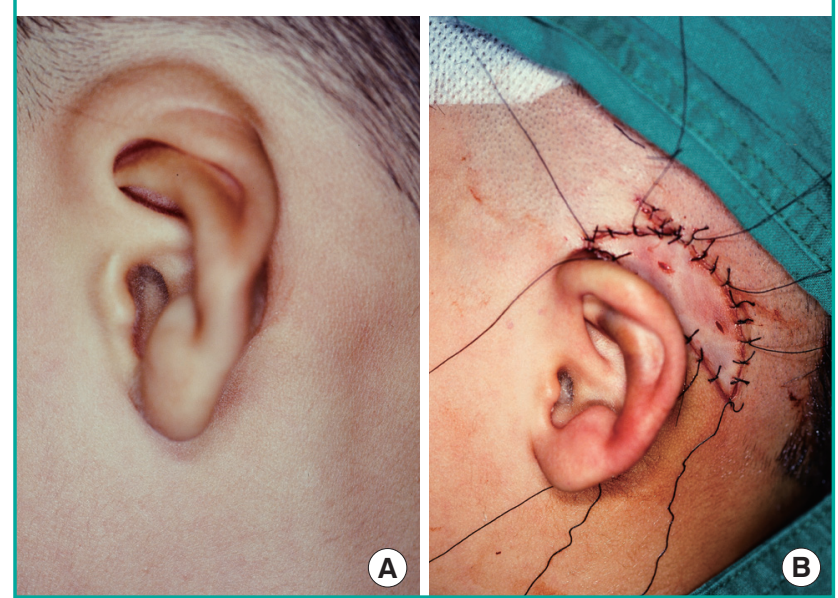


\section{RESULTS}

Among the 22 type I cryptotia patients, two whose deformities were corrected via autologous cartilage graft experienced reinvagination. Thus, the superior auricular sulcus had been made too shallow. Two patients whose deformities were corrected via Medpor implantation developed complications due to exposure. The 8 patients who had mild deformities did not develop any complications. Among the type II cryptotia patients, two who

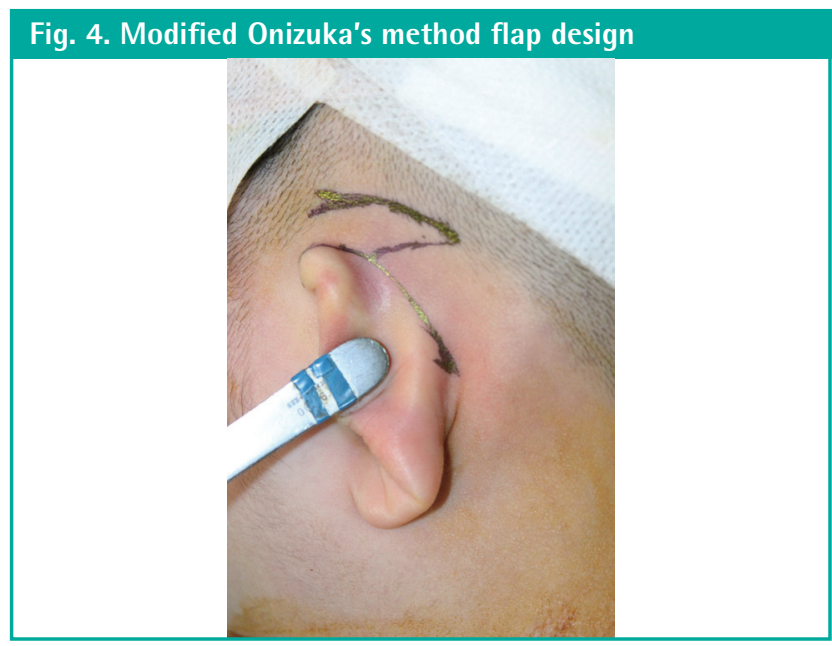

had received Medpor implantation grafts developed a hypertrophic scar, and the 10 with mild deformities did not develop any complications.

\section{DISCUSSION}

Cryptotia is quite rare among Europeans, but not among Asians.

Fig. 6. Nine-year-old type II unilateral cryptotic patient

(A) Preoperative view. The flap design was the modified Onizuka method. The cartilage deformities were corrected via cartilage graft from the cavum concha. (B) Findings at 4 months postoperatively.

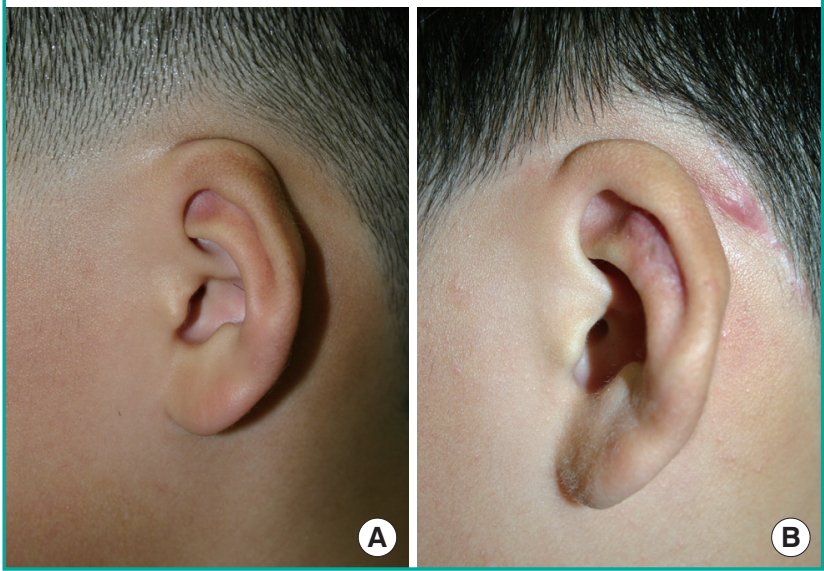

Fig. 5. Eleven-year-old type I bilateral cryptotic patient

$(A, B)$ Preoperative view. $(C, D)$ The flap design was the modified Onizuka method. The cartilage deformities were corrected via Medpor. $(E, F)$ Findings at six months postoperatively.
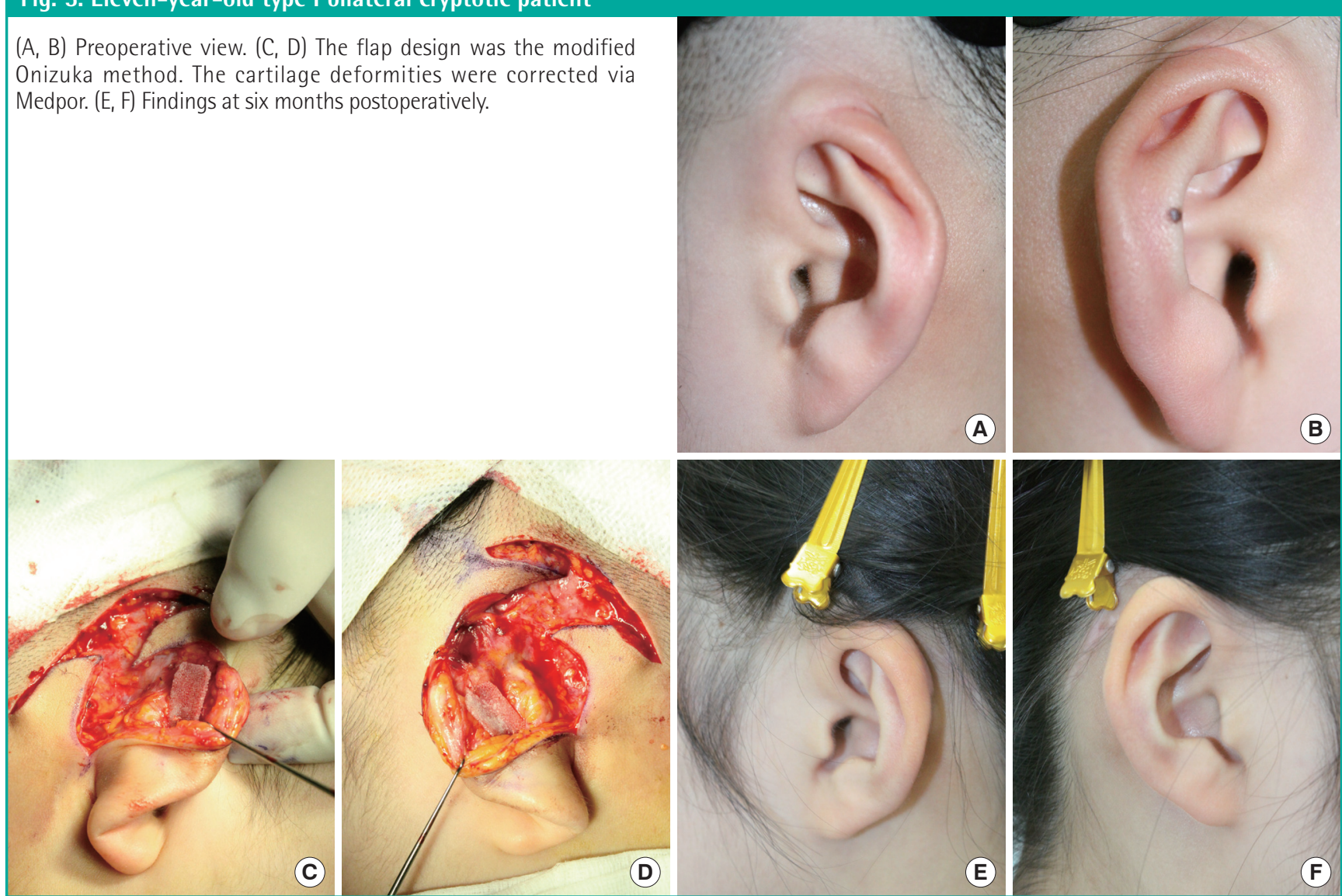
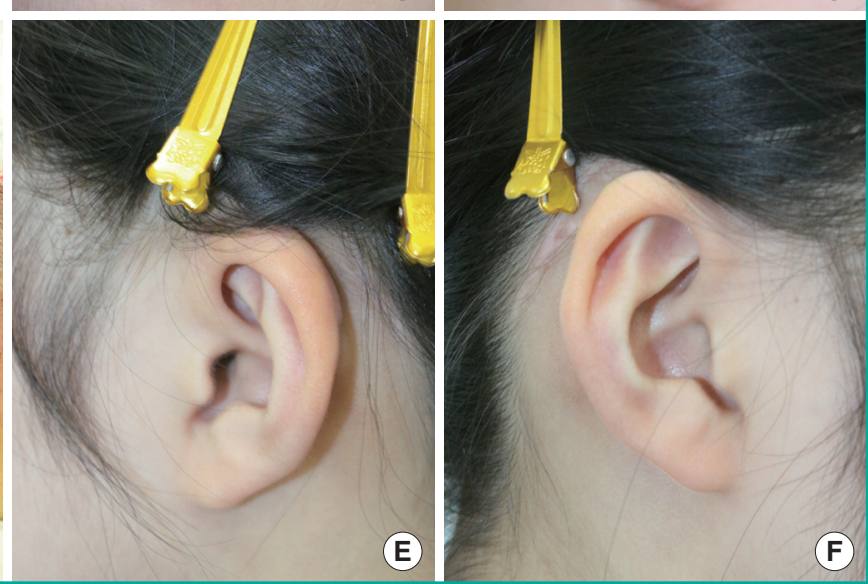
From the investigation conducted by Tanemura [4], Tachibana [5], and Fukuda [6], it can be speculated that the incidence of this abnormality in Japan might be one case in nearly every 400 births [7]. It is believed to be the same in South Korea. Cryptotia affects males more than females $(2: 1)$, and the right auricle more often than the left $(2: 1)$. There are more cases of unilateral cryptotia than bilateral cryptotia (7:3) [8]. The most common features of cryptotia are the invagination of the upper part of the auricle under the deformity of the auricular cartilage itself [2]. The cause of the deformity is unknown. Wreden proposed that cryptotia develops due to the anomalous insertion of the superior auricular muscle into the upper part of the helix rather than the eminentia triangularis [9].

Hirose classified cryptotia into two types, in accordance with the type of antihelix deformity and the state of the intrinsic auricular muscle. According to Hirose, there are considerably more cases of type I cryptotia than type II cryptotia [2]. These present authors decided that the cryptotia classification of Hirose was reasonable through our clinical experience.

Cryptotia can be managed through non-surgical treatment or surgical treatment. The non-surgical treatment or correction of cryptotia is carried out using a prosthesis. Many surgical techniques for the correction of cryptotia have been reported. The representative methods are the $\mathrm{V}-\mathrm{Y}$ advancement flap, rotation flap, transposition flap, and Z-plasty [1].

$\mathrm{Xu}$ et al. [10] introduced the surgical technique of correction of cryptotia with the square flap method in 2009, Kajikawa et al. [11] with the switched double-banner flap method in 2009, Sakamoto et al. [12] with the superior auricular myocutaneous flap in 2010, and Adams et al. [13] with the modified trefoil flap in 2011. Because it has recently been announced that cryptotia can be corrected via surgery, the authors are referring to recent research while doing their own studies to improve the surgical method and to decrease the complications.

Most cryptotic patients have auricular-cartilage deformities. The body of the antihelix and the superior or inferior antihelix are adhered together, and cryptotia cannot be successfully treated without resolving this adhesion. After removing the adhesion with a scalpel or scissors, a substance should be used as padding, to act as a splint. This will prevent the recurrence of adhesion and secure the substance in place and spread out.

In mild cases, the correction of a deformed cartilage is performed first through the incision of the cartilage deformity, and then by performing mattress sutures. In severe cases, the autologous cartilage and other substances are used as a splint after deformity correction in order to prevent recurrence.

In the case reported herein, the authors corrected the cartilage deformity of antihelical adhesion by cartilage graft from the ca- vum concha or Medpor. The Onizuka method is one of the best known methods of cartilage transplant, removing the bend of the cartilage by making multiple incisions at the back of the superior crus of the antihelix, and then collecting the largest cartilage and transplanting it into the cartilage of the antihelix to act as a splint. Cartilage graft from the cavum concha is used because it has a lower frequency of occurrence of inflammation and infectionMedpor implant, on the other hand, has the dangers of exposure and inflammation. If the injury is minimized by covering the insertion with a sufficient layer of muscle then through the patient's education, it may be used semi-permanently. As it is moderately robust, which makes it easier to handle during surgery, and has a number of microholes, it could be easy for the new blood vessels to grow in and to develop strong resistance to infection. Thus, it is considered to have high utility as a graft during the correction of cryptotia. It was recently reported that it can be widely used due to its advantages, such as a sufficient quantity for grafting, ease of shape maintenance, and lack of a need for a donor [14]. In this study, the type I cryptotia patients had severe cases of cartilage deformity. Thus, cartilage graft from the cavum concha or Medpor implantation was often performed on the type I cryptotia patients. Whether the cartilage deformity in type I cryptotia was severe or not was not significant statistically in this study. Further research and evaluation is thus needed. The research on the classification and surgical treatment of cryptotia must be continued to decrease its recurrence rate and complications.

The 34 cryptotia patients in this study with intrinsic anomalies and antihelical deformities were divided into the type I and II cryptotia groups. The results of the surgical treatment were compared. The type I cryptotia patients had more severe deformities than the type II cryptotia patients. Cartilage graft from the cavum concha and high-density polyethylene implants were used for the severe auricular-cartilage deformities.

Due to the anatomical characteristics of cryptotia, the management of the associated cartilage and skin deformities required consideration. Constructing an auriculocephalic sulcus and correcting the cartilage deformity at the same time can help prevent its recurrence.

These authors' hospital is currently researching better surgical methods and ways to prevent related complications based on the classification of cryptotia. Continued research concerning cryptotia is needed hereafter.

\section{REFERENCES}

1. Yotsuyanagi T, Yamashita K, Shinmyo Y, et al. A new operative method of correcting cryptotia using large Z-plasty. Br J Plast Surg 2001;54:20-4. 
2. Hirose T, Tomono T, Matsuo K, et al. Cryptotia: our classification and treatment. Br J Plast Surg 1985;38:352-60.

3. Kim JH, Jung YH, Lee KN, et al. Modified Onizuka's method for correction of cryptotia. J Korean Soc Plast Reconstr Surg 1996;23:451-60.

4. Tanemura T. Upon the pocket ear. Jibi-Inko-Ka 1935;8: 401-7.

5. Tachibana M. Upon the experience of treating the cryptotia. Jibi-Rinsho 1941;36:167-72.

6. Fukuda O. Otoplasty of cryptotia. Keisei Geka 1968;11:11725.

7. Ohmori S, Matsumoto K. Treatment of cryptotia, using Teflon string. Plast Reconstr Surg 1972;49:33-7.

8. Ohmori S, Takada H. Cryptotia. Aesthetic Plast Surg 1979; 3:15-28.

9. Marsh D, Sabbagh W, Gault D. Cryptotia correction: the post-auricular transposition flap. J Plast Reconstr Aesthet
Surg 2011;64:1444-7.

10. Xu JH, Wu WH, Tan WQ. Surgical correction of cryptotia with the square flap method: a preliminary report. Scand J Plast Reconstr Surg Hand Surg 2009;43:29-35.

11. Kajikawa A, Ueda K, Asai E, et al. A new surgical correction of cryptotia: a new flap design and switched double banner flap. Plast Reconstr Surg 2009;123:897-901.

12. Sakamoto Y, Nakajima H, Kishi K, et al. A new surgical correction of cryptotia with superior auricular myocutaneous flap. J Plast Reconstr Aesthet Surg 2010;63:1995-2000.

13. Adams MT, Cushing S, Sie K. Cryptotia repair: a modern update to the trefoil flap. Arch Facial Plast Surg 2011;13: 355-8.

14. Lee KC, Kwon YS, Heo J, et al. Otoplasty with high density polyethylene implant (Medpor(R)). J Korean Soc Plast Reconstr Surg 2009;36:167-73. 